\title{
Il faro dell'isola del Tino. Trasformazione di una struttura di difesa in riferimento per la navigazione
}

The Tino Island's lighthouse. Transformation of a defensive building to a reference for navigation

\author{
Fabio Borghini \\ Architect, Genoa, Italy, borghinifabio90@gmail.com
}

\begin{abstract}
The special and unique position of the Tino Island in the Gulf of La Spezia, with its complex orography, has strengthen his attitude of sighting, protecting and controlling the sea over centuries. Nothing can escape the view enjoyed from the top of the island, which on the clearest days catches a stretch of sea that goes from Livorno to Genoa. This was certainly noticed by the Republic of Genoa's engineers around 1600 , who probably built the current base of the lighthouse that still exists today. This tower was part of the program to strengthen the defense of the Ligurian's eastern coast. The structure, of which there are no detailed studies, has a remarkable typological similarity with the ruins of the only surviving coeval tower, Torre Scola, not far from Palmaria Island. It's not certain that the tower on Tino Island had an offensive character, because its extremely high position relative to the water level would be too difficult to shoot an enemy on the sea, but it is still possible to see defensive features that could be used if the structure had been besieged. What's certain is that his vocation of control over the sea was never lost, even if Napoleon wanted to transfer this facility to the nearby Palmaria Island. We owe its renovation to the Savoy government, in particular to King Carlo Alberto, who first wanted to build a lighthouse in 1839, adding a small tower to the Genoese structure. Then in 1884, the second lighthouse was built, higher than the first, which can still be seen today and that is still used for the light signals. These structures were added over time to house the lighthouse's staff. Today the island is closed to the public, as it is the property of the Italian Navy, and perhaps, thanks to this, it has kept intact the features of its architecture surrounded by nature, as it certainly was in the century of its construction.
\end{abstract}

Keywords: Landscape, costal tower, lighthouse, Liguria.

\section{Introduzione}

Possiamo leggere la particolarità dell' architettura difensiva dell'isola del Tino nel Golfo della Spezia tenendo ben a mente quello che Aldo Rossi affermava nel suo testo L'Architettura della Città a proposito del concetto di locus, definito come il rapporto singolare ed universale che esiste tra una certa situazione locale e le costrizioni in esso inserite all'intero della città. Certamente noi non ci troviamo di fronte ad uno spazio urbano, tutt'altro, siamo invece in un paesaggio insulare, fra $\mathrm{i}$ più preservati della penisola italiana. Ma ciò non toglie che si possa trasporre il concetto rossiano di locus all'interno del paesaggio. Nel caso dell'isola del Tino infatti si instaura una forte relazione fra la singolare architettura monumentale presente nella sommità dell'isola e il particolare 
contesto paesaggistico che la corona. L'interrelazione di architettura e paesaggio da sempre caratterizzano questo luogo, il quale grazie al reciproco raffronto acquisisce un valore in più che non è dato dalla sola e semplice somma dei due elementi dati. Nell'immaginario collettivo di chi fa esperienza di questo territorio è sedimentata l'idea di un'isola resa inseparabile dall'architettura che la sormonta e che a sua volta ne completa il profilo paesaggistico.

Ben consci dunque del locus presente sull'isola è interessante anche indagare e focalizzarsi sul fattore tempo, ovvero come questa fortificazione ha attraversato i secoli della sua storia e come si sia modificata tramite addizioni e ampliamenti successivi, il tutto senza mutare la sua forma originaria. Il nucleo generatore di cui si tenterà in seguito di correggere la datazione, è stato mantenuto ed è oggi monumento, ovvero portare di memoria del sito in cui sorge. Attitudine non sempre attribuita in passato a questi manufatti, visto che nel Golfo della Spezia altre torri costiere presumibilmente coeve a quella in esame sono state eliminate o hanno fortemente rischiato la demolizione a cavallo fra Ottocento e Novecento.

La forma, quindi, divenuta memoria surclassa la funzione originaria, e nel caso specifico della torre esagonale dell'isola del Tino, la fortificazione muta in faro. La struttura rimane, si arricchisce, e la funzione se pur simile varia. Si passa da un'architettura fatta per sorvegliare il mare ed avvistare il nemico, possibilmente rimanendo il più celata possibile, a complesso volto invece a farsi vedere e per segnalare il pericolo degli scogli affioranti presenti nel mare sottostante.

È sempre interessante l'esercizio di lettura della complessità architettonica di alcune costruzioni che hanno attraversato la storia. Ciò accade nel piccolo anche nel caso del Faro del Tino. Qui persino ad un occhio non esperto risulta evidente che la forma esagonale, che costituisce oggi la solida base della lanterna, sia di un'epoca radicalmente diversa rispetto al edificio a ferro di cavallo che le si addossa.

Risulta abbastanza chiaro quindi che la prima edificazione di questo complesso sia stata proprio tale torre, costruita sulla sommità dell'isola quasi certamente come punto di avvistamento sull'ampio tratto di mar Tirreno. L'isola del Tino è infatti l'affaccio più a sud del golfo della Spezia e più esposto verso il mare aperto. Va ricordato, come nota interessante, che nelle giornate particolarmente terse, grazie alla sua posizione favorevole, è possibile abbracciare con la vista la costa tirrenica da Livorno fino a Genova, comprese le isole dell' arcipelago toscano (Gorgona e Capraia) e la Corsica. La scelta del sito sembra sia stata ben ponderata dagli ingegneri militati genovesi, che decisero qui di insediare il primo presidio militare mai costruito nell'isola.

Non è però la prima opera di questa natura presente nel golfo della Spezia, infatti sin dal 1564 era presente un possente presidio militare, edificato più internamente nell'insenatura, meglio nascosto alla vista dei vascelli che tentavano di addentrarsi e capace di cogliere di sorpresa le incursioni nemiche. Questo era l'intento della Fortezza di Santa Maria, oggi completamente perduta e considerata insieme alla fortezza del Priamar di Savona e il Forte di Gavi, uno dei tre grandi avamposti militari di confine della Serenissima Repubblica di Genova. (Borghini, Maggiani, 2016) (Fig. 1).

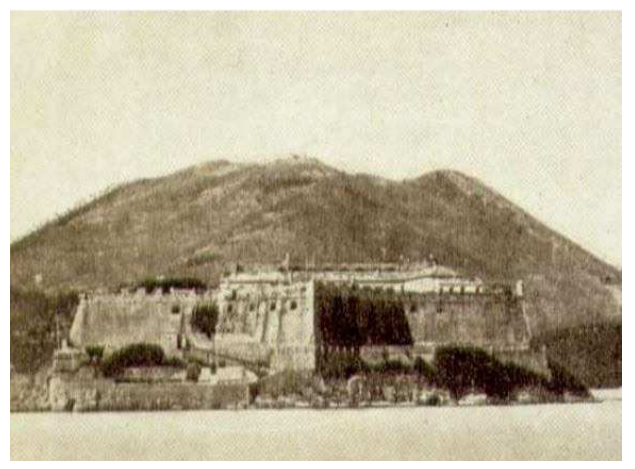

Fig. 1. Foto storica della Fortezza di Santa Maria.

Questa grande fortezza a forma di stella a 5 punte, figlia della trattatistica militare di tradizione rinascimentale fungeva da grande elemento deterrente ad un possibile attacco nel Levante ligure. Costruita durante il periodo cosiddetto el siglo de los Genoveses, epoca di fiorente legame con la Spagna e la Superba, di lì a poco risenti delle mire 
espansionistiche della corona spagnola nella penisola italiana. Il potente impero infatti per molto tempo cercò di accaparrarsi il controllo della Lunigiana e del golfo spezzino al fine di poter consegnare l'agognato affaccio al mare al Ducato di Milano. Così facendo si sarebbe garantito il diretto contatto via mare con l'altro possedimento iberico in territorio italiano, ossia il regno di $\mathrm{Na}-$ poli. Tramite la diplomazia, la mira espansionistica era notizia ben nota alla Repubblica, che ferita dal susseguirsi delle inadempienze finanziarie spagnole, cominciò a temere lo storico alleato (Mazzini, 1920).

Al prospettarsi di tale pericolo Genova decide di fortificare maggiormente il golfo, inizialmente chiedendo la consulenza di diversi esperti; per poi realizzare in tempo record fra il 1605 e il 1608 il complesso sistema di torri costiere. Di queste facevano parte la torre di San Giovanni Battista o della Scola presso l'isola Palmaria, di Sant'Andrea nell'odierna punta Pezzino e di San Michele nei pressi del paese di Cadimare per quel che concerne la costa occidentale mentre per ciò che riguarda la costa orientale del golfo venne eretta solo la torre dell'Oca Pelata, poi nota successivamente come torre di Santa Teresa (Cevini, 1984).

Dai resti dell'unica torre sopravvissuta al tempo, la Scola (Fig. 2), e dai disegni riportati per la torre di Sant'Andrea, notiamo che la forma comune a tutti queste opere era caratterizzata da una pianta poligonale regolare che si elevata in altezza con una muratura a scarpa per i 3/4 dello sviluppo verticale ed era coronata con una piazza d'armi in sommità, protetta da possenti troniere e da garitte angolari.

Questo sistema di torri che faceva capo alla Fortezza di Santa Maria venne perfezionato nel suo funzionamento dall'ingegnere francese Antoine Frédéric Flobert durante i suoi numerosi sopralluoghi per conto della Serenissima Repubblica di Genova. Ovviamente venne sancita come sede del Comando Superiore la Fortezza di Santa Maria la quale ogni giorno doveva avere collegamenti terrestri o marittimi con le strutture minori. Ciò sottolinea l'attenta cura nella realizzazione di queste opere intese quindi come un vero e proprio sistema difensivo capace di mantenere e garantire la protezione di un territorio morfologicamente complesso.

Successivamente ai rimaneggiamenti delle difese durante il Seicento, il territorio spezzino sarà coinvolto nel 1748 in una riorganizzazione delle fortificazioni sotto il controllo di Louis François Armand de Vignerot du Plessis Terzo Duca di Richelieu (1696-1788) e dei suoi collaboratori. Il Duca, noto generale dell'esercito francese, si distinse per le sua capacità militari nell'ambito della Guerra dei Sette anni e per questo venne insignito del prestigioso titolo di Maresciallo di Francia nel 1748. É noto il suo arrivo a Genova alla guida di un esercito che aveva l'obiettivo di liberare la città dall'occupazione austriaca ed è con molta probabilità che per riconoscimento gli venne affidato l'incarico di sovrintendere alle difese della Repubblica. Va ricordato infatti che ormai dal 1684, anno del bombardamento di Genova da parte della flotta di Luigi XIV, la repubblica è definitivamente ritornata sotto la sfera di influenza francese.

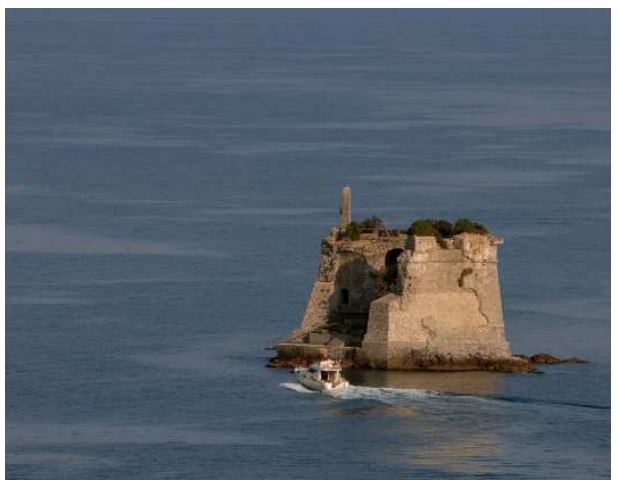

Fig. 2. Attuale immagine dei resti della Torre di Sant'Andrea.

Questo compito di sovrintendente alle difese, che riguardò anche il Levante, è ben documentato da una fitta rete di scambi epistolari fra gli accompagnatori del Duca e la capitale. Dalla Spezia giungono a Genova rapporti estremamente precisi circa gli spostamenti e i sopralluoghi del nobile ospite, senza tralasciare ovviamente le scelte e le migliorie che vengono ordinate per le varie fortezze presenti nel golfo (Archivio di Stato di Genova, Archivio Segreto, 2893). 
Dai rapporti siamo certi che i sopralluoghi del Duca non riguardarono la torre sull'isola del Tino $\mathrm{e}$ in nessuno dei documenti coevi viene mai menzionata l'esistenza di questa costruzione, ciò porterebbe a pensare che all' anno 1748 l'edifico non fosse ancora edificato. Allo stesso tempo però non vi è notizia circa l'intenzione di migliorare le difese approntando una nuova costruzione sull'isola.

\section{La datazione}

Non è possibile lanciarsi in ipotesi di datazione con il solo supporto delle fonti di archivio, risulta necessaria una analisi del manufatto e del suo sviluppo, possibilmente cercando di compararlo con altri segni simili presenti nel territorio.

Come già accennato, abbiamo la fortuna di poter ancora leggere con molta facilità il nucleo esagonale che oggi funge da base del faro. Questo ci dice già qualcosa circa la natura costruttiva della torre rispetto agli annessi successivi. Infatti questi ultimi costituiti da una muratura di carattere più edilizio difficilmente si omogenizzano con dei muri la cui funzione è principalmente militare e $\mathrm{i}$ cui spessori non consentono ampi margini di modificazione.

La torre infatti si identifica, oltre per la forma esagonale della pianta, anche per una possente muratura a scarpa di notevole spessore che si innalza per circa $8 \mathrm{~m}$. Questa è interrotta da un cordolo in pietra a sezione semicircolare che cinge la torre e che segnala la variazione di inclinazione della muratura, che si sviluppa perfettamente a piombo che corona l'intera struttura. Questo modo di concepire l'architettura difensiva non è per nulla lontano da quello utilizzato per la realizzazione della torre della Scola precedentemente citata, che ci porta a pensare appunto ad una realizzazione coeva delle due strutture (Borghini, 2017). È possibile approntare lo stesso raffronto con la Torre di Sant'Andrea che sorgeva sulla punta del Pezzino, di cui oggi ci rimangono solo i rilievi e le immagini dipinte e disegnate nell'Ottocento. Come le altre torri anche quella del Tino si concludeva in sommità con una piazza d'armi, oggi completamente coperta dalle strutture del faro. Sulla copertura, in coincidenza dei vertici dell'esagono erano poi alternate tre grandi garitte a forma pentagonale ancora oggi riconoscibili in quanto il loro volume va ad innestarsi vistosamente con la muratura a scarpa basamentale (Fig. 3). La loro posizione era ben codificata visto che ognuna è orientata secondo una precisa direttrice di visuale, ottenendo così un controllo quanto mai esteso sul mare.

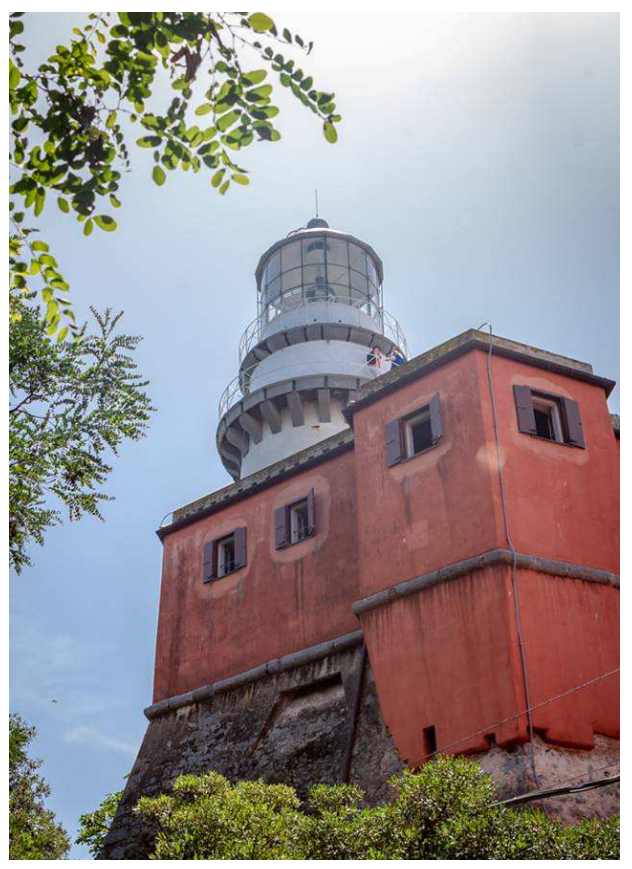

Fig. 3. Attuale immagine del Faro del Tino, da notare le evidenti forme militari della struttura oggi base della lanterna (Foto Roberto Celi).

La torre era priva di ogni bucatura, a meno dell'ingresso che si apre in direzione sud ed è oggi inglobato all'interno degli annessi del faro. Da qui poi ci si introduceva ad una grande sala coperta con una cupola in blocchi di pietra. Si presume che l'areazione fosse consentita dalle sei feritoie orizzontali che si aprono lungo ognuno dei sei lati dell'edificio, di cui non si è certi se avessero anche una funzione militare qualora la torre fosse stata presa d'assalto (Fig. 3). All'interno si presume vi fossero gli alloggi per la guarnigione di cui poco si sa. Attualmente è presente un forno a legna, di cui però non si ha la certezza della sua originalità, ma sembra piuttosto una aggiunta successiva di cui non si può dare una datazione certa. 
L'organizzazione interna formata da un unico vano voltato rimanda nuovamente all' affinità con la Torre della Scola, la quale sebbene diroccata ci suggerisce una medesima organizzazione.

Tutto ci porta a pensare che la fortezza del Tino sia coeva a quelle realizzate negli anni fra 1606 e il 1608 dalla Repubblica di Genova. Ciò però non è possibile da certificare con i documenti di archivio e le varie testimonianze successive, che non menzionano mai la torre in esame.

Questo accade anche per i documenti che riguardano il sopralluogo del Duca di Richelieu del 1748. Anche qui nei rapporti inviati a Genova non si fa mai menzione della struttura sull'isola del Tino.

Ci viene però in soccorso la cartografia coeva, dove invece la torre è segnalata. Dagli archivi cartografici dell'Archivio di Stato di Genova, consultabili on-line, è possibile rinvenire la presenza del manufatto in svariate carte riferibili alla prima metà del Settecento. Una di queste venne redatta dal cartografo ufficiale della Repubblica, il colonnello Matteo Vinzoni (1690-1773), proprio nel 1748 e dedicata come riportato sulla stessa mappa all'insigne ospite francese: "La Riviera di Levante, Stato di Genova ed altri confinati. Dedicata all'impareggiabile merito di S. Eccellenza il signor Luiggi Armando Duplessis, duca di Richelieu, pari di Francia, cavaliere dell'Ordine del Re, primo Gentiluomo della di Sua Maestà Christianissima".

Sorge spontanea quindi il perché tale struttura non risulti nei documenti. L'unica risposta che oggi ci può venire in mente è forse derivata alla funzione che questa doveva avere e che con molta probabilità non era legata strettamente alla pratica militare, ma solo all'avvistamento e alla segnalazione del nemico. Ed forse per questo che ad esempio non rientra all'interno del programma di potenziamento delle difese del golfo spezzino di metà Settecento.

L'assenza dai documenti scritti, dalle rendicontazioni, dai censimenti delle truppe e da tutto quanto è possibile consultare presso l'Archivio di Stato ha portato a pensare che la datazione dell'opera fosse da riferirsi all'età napoleonica.
Con molta probabilità per via di un'ingenua considerazione legata al fatto che una corposa documentazione dello stato delle opere di difesa del golfo la dobbiamo al precisissimo lavoro di rilievo del promontorio che va da Riomaggiore a Porto Venere, realizzato dalla brigata di topografi francesi negli anni 1809-1811 (Rossi, 2008).

Il raggruppamento di militari guidati dal colonnello Pierre Antoine Clerc, vennero incaricati dallo stesso Napoleone di approntare un rilievo dello stato dei luoghi di questo territorio, in vista della volontà di realizzare un grande arsenale nelle insenature delle Grazie, del Varignano e della Castagna.

I soldati francesi sono quindi i primi a restituirci una mappa scientificamente corretta dell'isola del Tino e di ciò che allora conteneva, non solo realizzando una planimetria generale, ma andando anche a registrare con estrema precisione tutti i i prospetti degli edifici segnalati in pianta. È grazie a questa immensa opera che noi oggi conosciamo l'originario aspetto della torre del Tino prima della creazione del faro (Fig. 4). É da queste restituzioni che con molta probabilità si è incorsi nell'errore di datazione ancora oggi riscontrabile e che fa risalire la fondazione all'età napoleonica. Ma come si è precedentemente notato tale ipotesi è smentita dalle ricognizioni sulle cartografie storiche.

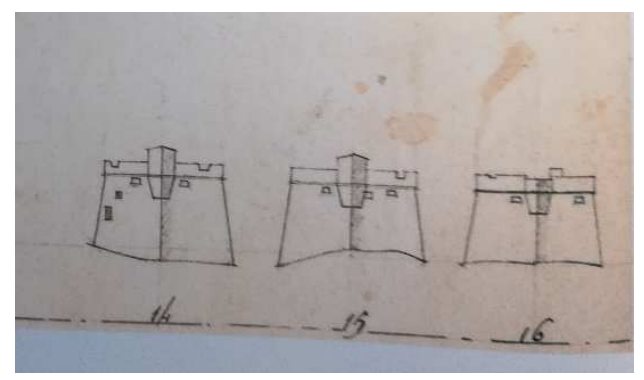

Fig. 4. Particolare del foglio n. 18 della Brigata topografica di Clerc (da Rossi, 2008, p. 166).

Questo ambivalenza delle datazioni è riscontrabile anche a livello burocratico nel decreto di vincolo che insiste sul manufatto. Il documento, facilmente scaricabili dal sito liguriavincoli.it, riporta infatti una relazione datata 30 agosto 2012, 
nella quale si sottolinea che "il progetto e la realizzazione di un forte sulla sommità dell'isola del Tino $[\ldots]$ risalgono al periodo della dominazione francese".

In calce al testo citato vi è però riportato il decreto precedente, datato al 21 ottobre 1973, molto meno esaustivo, ma che ci dice che la torre "costituisce un notevole esempio di costruzione militare del secolo XVI".

La questione della datazione di questa struttura rimarrà sicuramente aperta, ma quello che possiamo sicuramente affermare con certezza è che la sua realizzazione si deve per forza di cose collocare in un periodo che va dagli inizi del Seicento alla prima metà del Settecento. La forbice temporale è molto vasta, ma certamente si può e si deve escludere l'ipotesi di collocare l'opera nel periodo Napoleonico.

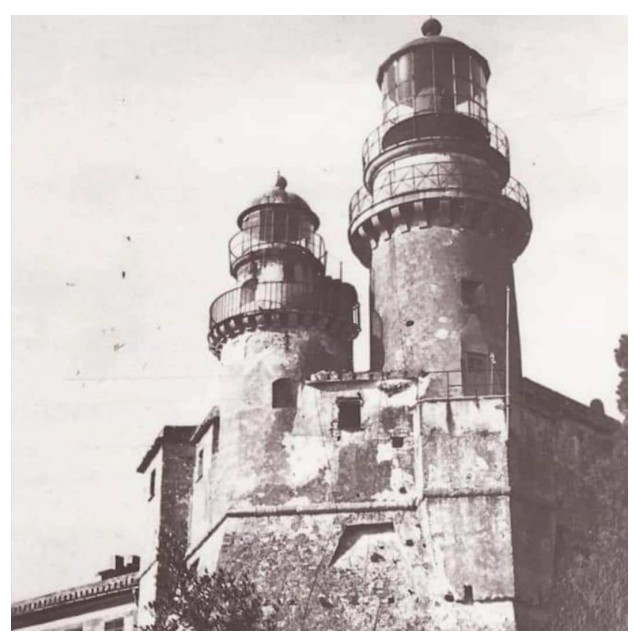

Fig. 5. Immagine storica delle due lanterne ancora oggi presenti nel complesso.

Le vicende successive al passaggio dei cartografi napoleonici, e partono dalla restaurazione e dalla dominazione sabauda, ci dicono che per volere di re Carlo Alberto venne edificato il primo faro nel 1839 , ancora oggi visibile, e collocato in uno dei vertici dell'esagono fra le due garitte. Il funzionamento della lanterna avveniva tramite la combustione di olio vegetale, successivamente sostituito dal carbone. Di lì a poco, nel 1884, venne innalzata la seconda torre (Fig. 5), quella che ancora oggi è utilizzata, più alta della prima. Inizialmente questa veniva alimentata elettricamente da due macchiane a vapore. Sappiamo però che nel 1912 l'impianto venne sostituito con uno ai vapori di petrolio, per poi essere elettrificato in tempi ancora più recenti, fino alla completa automazione nel 1985.

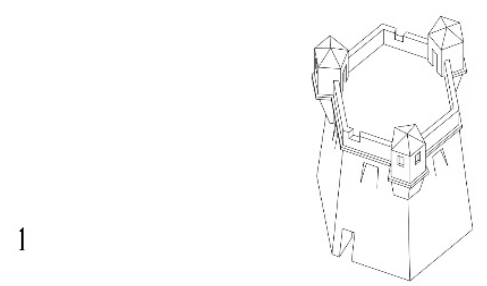

2
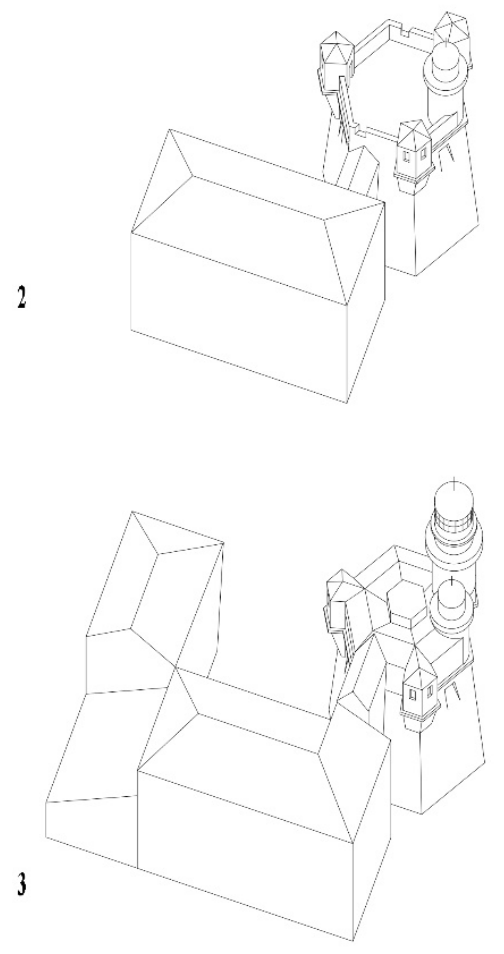

Fig. 6. Ipotetica ricostruzione delle tre principali fasi costruttive del complesso del Faro del Tino. 1) periodo di prima edificazione ricavabile dalla documentazione $\mathrm{Na}$ poleonica (vedi fig. 4); 2) fase della costruzione del primo faro nel $1839 ; 3$ ) assetto attuale pressoché inalterato dalla costruzione del secondo faro nel 1884 . 


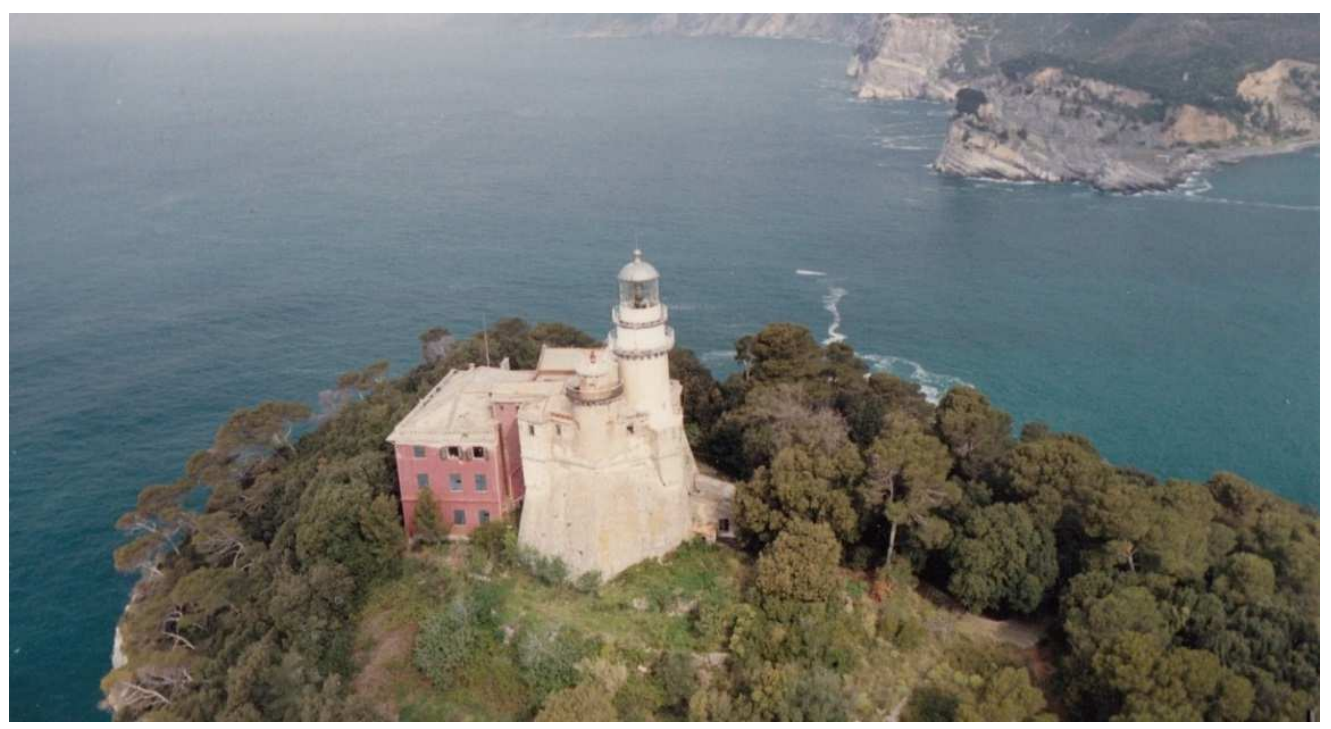

Fig. 7. Immagine storica del complesso del Faro del Tino all'interno del paesaggio costiero di Porto Venere. Dalla fotografia aerea è possibile notare la preesistenza storica della torre esagonale e la sua rilevanza monumentale rispetto alle edificazioni successive.

\section{Conclusioni}

Il lento trascorrere del tempo ha portato ad un inevitabile cambiamento dell'immagine che questa torre che aveva all'interno del sistema di difesa del Golfo della Spezia. Questo processo ha però incrementato il rapporto fra la stessa $\mathrm{e}$ il paesaggio che la circonda, legando architettura e contesto in un rapporto simbiotico e indivisibile, riconosciuto anche a livello internazionale grazie all'inserimento di questi luoghi all'interno del World Heritage List dell'UNESCO nel 1997 con il riconoscimento del sito "Porto Venere, Cinque Terre e Isole (Palmaria, Tino e Tinetto)".
Certi dunque del fatto che la torre esagonale dell'isola del Tino, oggi parte del complesso del Faro, non appartiene al periodo napoleonico. E consci che una sua datazione univoca è ancora difficile da collocare nell' arco di tempo individuato. Possiamo però sicuramente affermare che la sua posizione all'interno del riconosciuto paesaggio culturale dell' arcipelago di Porto Venere", è oggi quantomai rilevante. L'interazione di secoli fra uomo e natura sono passati anche per questi luoghi, lasciando un segno che noi oggi riconosciamo quale elemento qualificante di questo territorio, che è bene approfondire maggiormente al fine di giungere ad un migliore livello di consapevolezza dei manufatti che qui "abitano" da molto tempo.

\section{Bibliography}

Bosco, G. (1998). Progetti integrati per le antiche fortificazioni costiere, La Spezia-Porto Venere.

Braudel, F. (1986). Mediterraneo. Lo spazio e la storia, gli uomini e la tradizione, Bompiani, Milano.

Carrozzi, F. (1978). "La Torre Scola”, La Spezia, 1-2, 1978.

Caselli, C. (1998). La Spezia e il suo Golfo - Notizie storiche e scientifiche, Ristampa anastatica, Luna Editore, La Spezia.

Cevini, P. (1984). La Spezia, Sagep, Genova.

Faggioni, E. "La Torre Scola”, in La Nazione (19 July 77). 
Faggioni, G. (2008). Fortificazioni in provincia della Spezia - 2000 anni di architettura militare, Ritter Ed., Milano.

Faggioni, G. (2010). Le fortificazioni del Levante ligure: castelli e torri fra terra e mare. Fidenza, pp. 24-30, 137.

Fara, A. (1975). Funzione militare, architettura e urbanistica dell'Ottocento a La Spezia - Recupero di Domenico Chiodo, Firenze.

Formentini, U. (1934). “Monumenti di Porto Venere”, in Memorie dell'Accademia Lunigianese di Scienze, La Spezia.

Forti, L.C. (1992). Fortificazioni e ingegneri militari in Liguria: 1684 - 1814, Genova.

Forti, L.C. (1971). Le fortificazioni di Genova, Genova.

Giustiniani, A. (1854). Annali della Repubblica di Genova, illustrazioni di G. B. Spotorno. Genova. In Liber lurium Repipublicae genuensis (Historiae Patriae Monumenta), Torino, tom. I.

Marmori, F. (1968). Fortificazioni nel golfo della Spezia, Genova.

Marmori, F. (1973). Provincia di La Spezia. In I castelli della Liguria II, Bona, E.D., ed., Genova, pp. 667-672.

Marmori, F. (1998). "La fortezza di Santa Marian nel Golfo della Spezia", Memorie dell'Accademia Lunigianese di Scienze "Giovanni Cappellini”, LXVI. La Spezia.

Mazzini, U. (1920). "Il progetto genovese di scaricare la Magra nel Golfo", Memorie dell'Accademia Lunigianese Giovanni Cappellini, II, La Spezia.

Minola, M. (2006). Castelli e Fortezze di Liguria: un affascinante viaggio tra storia e architettura, Genova.

Minola, M. (2009). Fortificazioni in Liguria dal XVIII sec. alla Grande Guerra, Genova, pp. 190-192.

Quaini, M.; Vinzoni, M., eds. (1983). Pianta delle due Riviere della serenissima Repubblica di Genova divise ne' Commissariati di Sanità. Matteo Vinzoni, Sagep, Genova.

Quaini, M.; Costa Restagno, J. (1986). Carte e cartografi in Liguria, Sagep, Genova.

Rossi, L., ed. (2008). Napoleone e il Golfo della Spezia - Topografi francesi in Liguria tra il 1809 e il 1811, Silvana Editoriale, Milano.

Semino, M. (1985). "La Torre Scola all'Isola Palmaria. In Rivista di Studi Liguri, anno LI, n 1-3 Gen- Sett.”, Atti del convegno I liguri dall'Arno all'Ebro. Albenga 4-8 Dic 1982, pp. 149-165.

Vinzoni, M. (1773). Il Dominio della Serenissima Repubblica di Genova in terraferma, Genova, in www.e-corpus.org. 